\title{
In vitro Tomato Fruit Cultures Demonstrate a Role for Indole-3-acetic Acid in Regulating Fruit Ripening
}

\author{
Jerry D. Cohen \\ Horticultural Crops Quality Laboratory, Beltsville Agricultural Research Center, Agricultural Research \\ Service, U.S. Department of Agriculture, Beltsville, MD 20705-2350
}

Additional index words. auxin, Lycopersicon esculentum, organ culture, phytohormones, tissue culture

\begin{abstract}
An in vitro system was used for the production of tomato (Lycopersicon esculentum) fruit in culture starting from immature flowers. This system produced small parthenocarpic (seedless) fruit in response to $10^{-4} \mathrm{Mindole-3-acetic} \mathrm{acid} \mathrm{(IAA)}$ supplied in the medium. Other auxins, auxin conjugates and antiauxins tested were not effective or produced markedly fewer fruit. Additional IAA supplied to the fruit culture media before breaker stage resulted in an increase in the time period between breaker and red-ripe stages from 7 days without additional IAA to 12 days when $10^{-5} \mathrm{M} \mathrm{IAA}$ was added. These results suggest that significant changes in the ripening period could be obtained by alteration of auxin relationships in tomato fruit.
\end{abstract}

There are five classes of generally recognized plant hormones that control plant growth and development throughout the life cycle of the plant. By far the most studied plant hormone in relation to fruit ripening and postharvest storage has been ethylene. Although we know much less about the other hormones in relation to fruit development and ripening, the utility of numerous agricultural sprays and practices suggest that the complex series of reactions of ripening fruit are controlled by an interplay of several classes of these plant hormones (Bangerth, 1983). In general, a relationship can be envisioned where ripening is controlled by a balance among several factors, but the details of such interactions are not well established.

An important goal of our research program has been to develop methods for understanding the hormonal relationships in fruit during development and ripening. Of particular interest to us was the possible alteration of ripening by auxins. As stated by Brady (1987), "The hypothesis that endogenous auxins play roles as ripening inhibitors is attractive... Research in this area has been hampered by the difficulty of equating responses to externally applied regulators with endogenous control by the growth regulator, by the interactions of the different regulators, and by analytical difficulties..." An additional difficulty encountered in studying the role of auxins in fruit results from the treatment itself. Treatment of whole fruit is often difficult due to problems of entry and stability. Because of the low surface to volume ratio, high treatment concentrations are often required to increase internal levels significantly (Frenkel and Dyck, 1973) and such levels approach the concentrations that induce ethylene formation.

Fruit tissues have changing levels of IAA throughout their

Received for publication 16 Nov. 1995. Accepted for publication 7 Dec. 1995. This work was supported in part by grants from the U.S.-Israel Binational Agricultural Research and Development Fund (BARD, US-2498-94) and the U.S. Dept. of Energy (DE-AI02-94ER20153). The technical assistance of JoEtta Hubbard and Dia Newman is gratefully acknowledged. For discussions that resulted in this work and for their advice on the manuscript, the author thanks Janet P. Slovin and James R. Dunlap. Indole-3-acetyl-1-0- $\beta$-D-glucose and 4-Cl-IAA were from D. Keglevic and V. Magnus, respectively, Ruder Boskovic Institute, Zagreb, Croatia. Indole-3acetyl-e-N-L-lysine was from T. Kosuge, Univ. of California, Davis. Mention of a trademark, proprietary product, or vendor does not constitute a guarantee or warranty by the U.S. Dept. of Agriculture and does not imply its approval to the exclusion of other products or vendors that may be suitable. The cost of publishing this paper was defrayed in part by the payment of page charges. Under postal regulations, this paper therefore must be hereby marked advertisement solely to indicate this fact. developmental and ripening process (Buta and Spaulding, 1994; Miller et al., 1987; Miller, 1990). The levels of IAA appear to be related to ripening in two different ways in different fruits. The first pattern is shown by stone fruits such as peach, where IAA levels increase during the period of fruit growth, drop to a lower level during maturation, and finally increase dramatically during the period just preceding the ethylene burst at ripening (Miller et al., 1987). The data from peach support the long-held notion that very high IAA levels induce ethylene production during fruit ripening.

A second pattern is shown, however, by studies of auxin levels relative to ripening in tomato fruit. In tomato, the levels of IAA drop throughout fruit expansion (Buta and Spaulding, 1994) to very low levels. Such low levels are only rarely seen with other plant tissues and it is these auxin-deficient tissues that carry out the program for the terminal events of fruit ripening.

In addition to changes in the levels of IAA, tomato fruit also changes in its capacity to metabolize IAA during growth and ripening (Catalá et al., 1992, 1994; Riov and Bangerth, 1992; Slovin and Cohen, 1993). Thus, the fruit is undergoing significant changes in both steady-state levels of IAA and in the metabolic processes that regulate the conjugation and degradation of IAA.

In studies on fruit physiology and, in particular, the hormone biochemistry of fruit tissues, researchers have traditionally been hampered by the size or bulk of the organ as well as the complexity of interactions occurring within such plant materials. Size limitations have often been overcome by the use of tissue pieces and slices. Unfortunately, the dangers of extrapolating from results obtained with wounded and rapidly repairing tissue to what is occurring in a bulky fruit in a maintenance phase as it moves toward ripening and senescence, have been only occasionally noted (Brady, 1987; Gouble and Soudain, 1993). The use of tissue discs have some advantages over whole fruit in terms of reduction of entry problems and tissue volume, but problems result from IAA oxidation at the cut surface (Castillo, 1986; Catalá et al., 1994) and a variety of wound effects including wound ethylene formation (Campbell et al., 1990; Catalá et al., 1992; Gouble and Soudain, 1993). In addition, tissue discs only undergo a limited program similar to the early events of the ripening period, show inconsistent effects upon treatment with physiological levels of IAA (Yunovitz and Gross, 1994), and show an altered pattern of ethylene evolution (Campbell et al., 1990; Gouble and Soudain, 1993). Thus, for studies of hormonal regulation of ripening, tissue discs may not accurately represent the normal ripening process as it occurs in the whole fruit. 
Studies on the metabolism of IAA as well as other hormones has been aided by the ease of labeling of small seedlings grown on agar in sterile culture (Normanly et al., 1993; Wright et al., 1991). Extension of such labeling techniques to fruit tissue presented significant problems. For example, wounded tissue has high peroxidative activity, which is known to degrade IAA (Kokkinakis and Brooks, 1979; Catalá et al., 1994). Thus, when using tissue sections, Yunovitz and Gross (1994) found no consistent effects of IAA until applications reached a level estimated to be 5000 fold the endogenous level (Buta and Spaulding, 1994). As shown by Catalá et al. (1994), twelve fold increases in peroxidases/IAA oxidase occur in cut tomato discs within the first $24 \mathrm{~h}$ post excision. These peroxidases account for the bulk of the decarboxylative IAA metabolism reported in such tissues and probably account for the discrepancy in the lack of effectiveness of IAA with discs, as cited above, and the small observed effect of IAA on delaying the ripening process or the initiation of ripening found with intact tomato fruit (Abdel-Kader et al., 1966; Babbitt et al., 1973; McGlasson et al., 1978). Of critical importance for studies of hormonal metabolism in fruit is the observation that the decarboxylative IAA metabolism noted in cut tissues does not occur in intact fruit (Catalá et al., 1992, 1994; Normanly et al., 1995).

Early studies by Nitsch (1951) showed that mature floral organs of tomato could be cultured and mature fruit obtained. More recently, Mukai (1985), Ishida (1991) and Ishida et al. (1993) have used a similar approach to study fruit ripening in vitro. For hormonal studies, it is important to use a system where large sample numbers can be obtained, and simple hormone treatments can be applied in the absence of developing seeds, which are known to produce hormones themselves (McGlasson et al., 1978). By using immature flowers grown under standard culture conditions, we show that a model parthenocarpic system has utility for studying the effect of phytohormones on fruit ripening.

\section{Materials and Methods}

'Ailsa Craig' tomato plants were grown in a greenhouse in 25$\mathrm{cm}$ pots containing a commercial soil and peat mixture. Soil moisture was maintained using an automated drip irrigation system. When the plants were mature and in full bloom, flowers were harvested using a sharp razor blade leaving $1 \mathrm{~cm}$ of stem attached. Immediately upon harvesting, the flowers were placed in ice cold water until all the flowers were harvested and brought to the laboratory. Flowers were then surface sterilized by treatment for $10 \mathrm{~min}$ in $400 \mathrm{ml}$ of a $10 \%$ solution of commercial bleach containing about $200 \mu \mathrm{l}$ of Tween 80 , then rinsed on a nylon screen with sterile distilled water and maintained in aseptic conditions. In a laminar flow hood, the flowers were prepared for planting by cutting the stem to about $1 \mathrm{~mm}$ in length and sorting by size. The planting medium consisted of Murashige-Skoog media salts (Sigma, St. Louis), Murashige-Skoog vitamins (see Gamborg and Shyluk, 1981), 3\% sucrose (Serva Biochemicals, Paramas, N.J.), 1\% Phytagar (Gibco/BRL, Life Technologies, Grand Island, N.Y.) and $10^{-4}$ M IAA (Sigma). The media were adjusted to $\mathrm{pH} 5.7$ before the addition of the agar. In some experiments other compounds were substituted for IAA, as noted below. In most experiments, the medium was sterilized in bulk with hormones added and distributed (about $20 \mathrm{ml} /$ tube) to $50 \mathrm{ml}$ polystyrene disposable centrifuge tubes (Corning, Corning, N.Y.). Cultures were maintained at 25C in a constant temperature room with continuous light at $25 \mu \mathrm{mol} \cdot \mathrm{m}^{-}$ ${ }^{2} \cdot \mathrm{s}^{-1}$ provided by cool white fluorescent lamps.

In experiments where a large number of different media were used, a slight modification of the above procedure was used. Medium without hormone was heated to melt the agar and $15 \mathrm{ml}$ poured into a $25-\mathrm{mm}$ dia. $\times 95-\mathrm{mm}$ shell vial containing $1 \mathrm{ml}$ of a concentrated stock hormone solution. Vials were then closed with a KimKap top (Kimax, Toledo, Ohio) before autoclaving. Compounds tested were: IAA, IAA ethyl ester, indole-3-butyric acid, 1naphthyleneacetic acid, phenylacetic acid and IAA-L-aspartate (Sigma), IAA-L-alanine, IAA-L-phenylalanine, 2-(p-chlorophenoxy)-2-methylproprionic acid and 2,4-dichloro-phenoxyacetic acid (Aldrich, Milwaukee, Wis.); N-(1-naphthyl)phthalamic acid (Pfaltz and Bauer, Waterbury, Conn.); methyl-2-chloro-9hydroxyfluorene-(9)-carboxylate, (CelaMerck, Ingelheim am Rhein, Germany); IAA-1-0-glucose and 4-Cl-IAA, IAA-L- $\varepsilon$-lysine and IAA-myo-inositol (mixed isomers, synthesized according to Nowacki et al., 1978)

In some experiments, $200 \mu \mathrm{l}$ of a solution of $10^{-3} \mathrm{M}$ IAA in water was added at specific periods after planting on IAA containing medium. The solution was pipetted into the agar at the edge of the tube using a Gilson micropipetor. Care was taken that no solution ran across the top of the agar and contacted the growing fruit. Experimental groups of 6 treated flowers and 6 controls were selected from larger plantings that were scheduled at 5 day intervals. When the first group reached breaker stage (day 62), all subsequent sample sets were treated with IAA. In this way, we generated a group of treatments and controls that were 5 days before breaker, 10 days, 15 days, etc. The whole experiment was carried out twice and the data are for the combined mean data $\pm \mathrm{SE}$ ( $n=12$ for treatments and $n=24$ for controls).

Analysis of free IAA was done according to Chen et al. (1988). Briefly, IAA was extracted in 70\% isopropanol/0.2 $\mathrm{m}$ imidazole buffer (v/v), pH 7.0 containing ${ }^{3} \mathrm{H}$-IAA and ${ }^{13} \mathrm{C}_{6}$-IAA as tracer and internal standard, respectively. The IAA was then purified on a Fisher PrepSep amino SPE column and on $\mathrm{C}_{18}$ HPLC on a Phenomenex UltraCarb $5 \mathrm{~cm} \times 4.6 \mathrm{~mm}$ column using $22 \%$ methanol : water plus $1 \%$ acetic acid as solvent. The IAA sample was then methylated (Cohen, 1984) and analyzed on a Hewlett Packard 5890 gas chromatograph/5971a mass selective detector using a J\&W DB-1701 capillary column (J\&W, Folsom, Calif.). Other details are described in Tam et al. (1995). Data are the mean + SE of three replicate fruit analyzed for each treatment.

\section{Results}

Designing the culture system. In the initial screen, flowers used for culture were selected at three stages of development based on size (3-4 mm, 5-6 mm, and 7-8 mm). All developmental stages tested formed fruit, but only on medium containing IAA (data not shown). Only one sample growing on no hormone set fruit, and upon dissection this fruit was shown to contain a small number of seeds. A larger study $(n=24)$ was then conducted using 3-4 mm flowers with and without IAA. Only the IAA treated flowers produced fruit, and these fruit did not contain seeds (Fig. 1). In addition to IAA, 15 other compounds were tested for their ability to support fruit development using this model system. None of the auxins, auxin conjugates, or antiauxins gave results comparable to IAA. IAA-alanine and IAA-aspartate each produced 1 fruit out of 6 flowers, while IAA treatment resulted in fruit developing from each of the 6 flowers planted on IAA media (data not shown). None of the other compounds tested produced fruit. Thus, the naturally occurring auxin, IAA, was used for all additional studies.

Based on these results, a larger scale experiment was designed to determine if significant numbers of such in vitro fruit could be produced using 3-4 mm sized flowers. An undergraduate student 


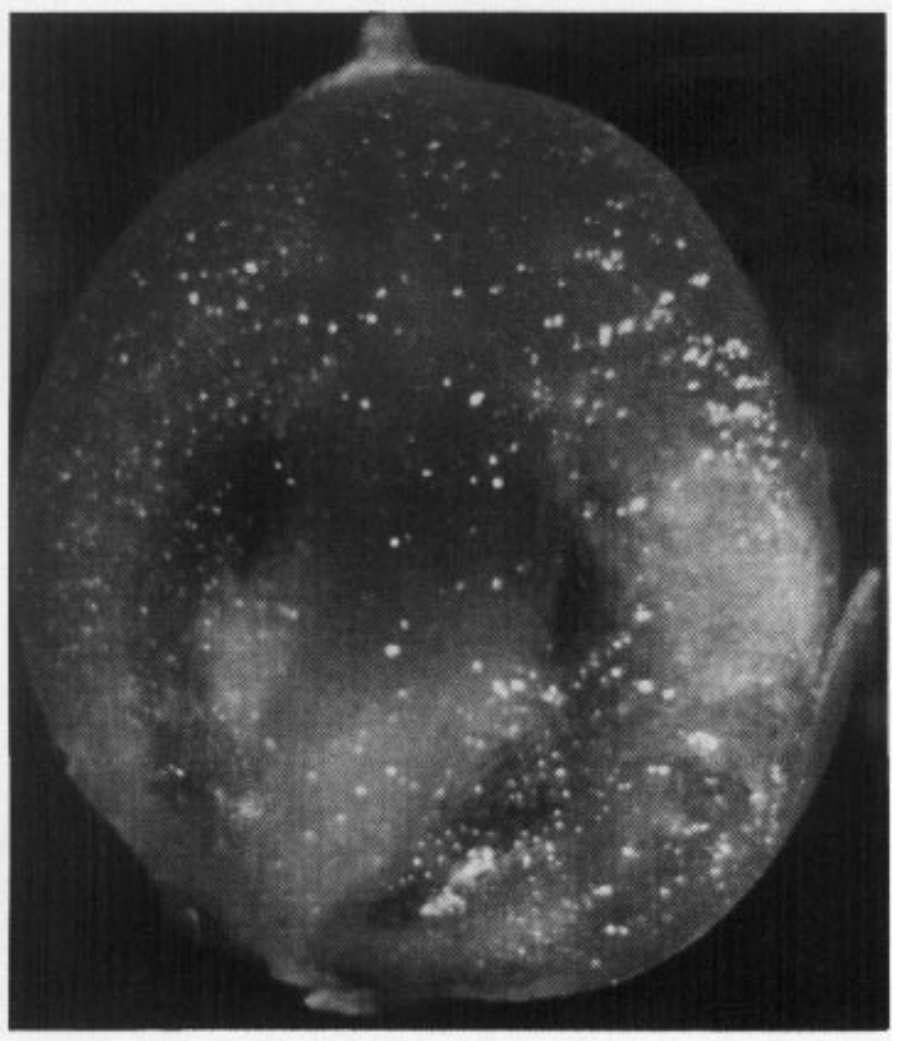

Fig. 1. Cross section through an in vitro grown 'Ailsa Craig' tomato fruit showing lack of seeds. White dots in photo are an artifact of the above stage lighting; photomicrograph is of a cross section through a $1.5-\mathrm{cm}$-diameter fruit.

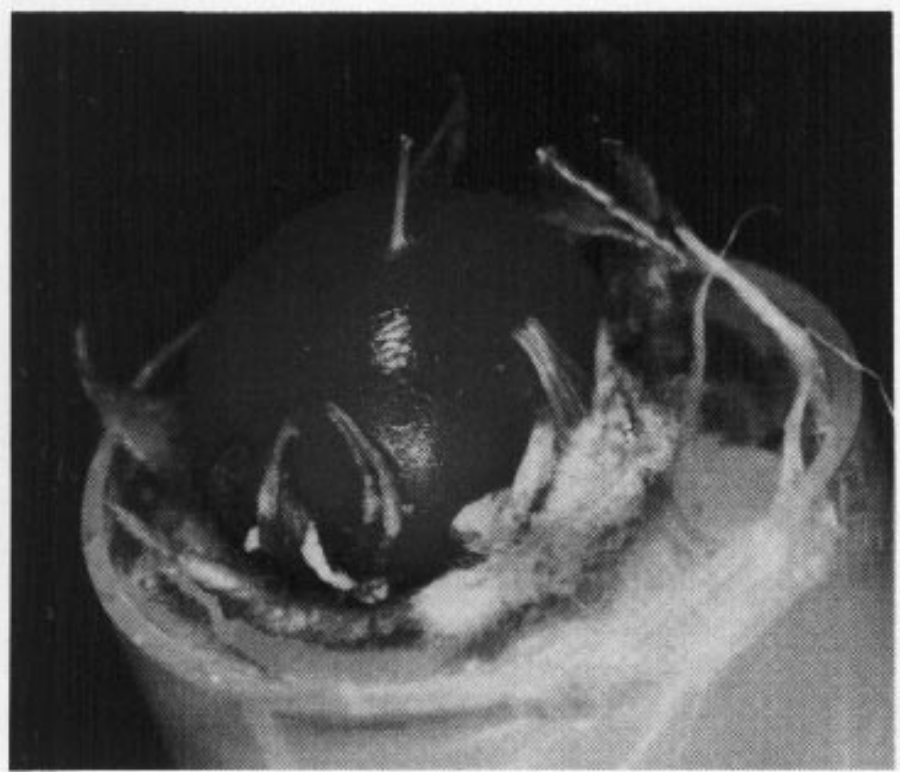

Fig. 2. Intact in vitro grown tomato at the red-ripe stage. Flower bud was planted on agar containing Murashige and Skoog medium with IAA in a $50 \mathrm{ml}$ sterile disposable centrifuge tube. The top portion of the tube was cut away before the photograph was taken (actual fruit size $\approx 1.5 \mathrm{~cm}$ in diameter).

was trained in this procedure and could easily produce more than 50 flower cultures per day. From a series of experiments where 500 flowers were planted on IAA media, contamination and flower death resulted in a loss of 43 flowers $(8.6 \%)$. All of the remaining flowers produced fruit, although 49 fruit (9.8\%) prematurely

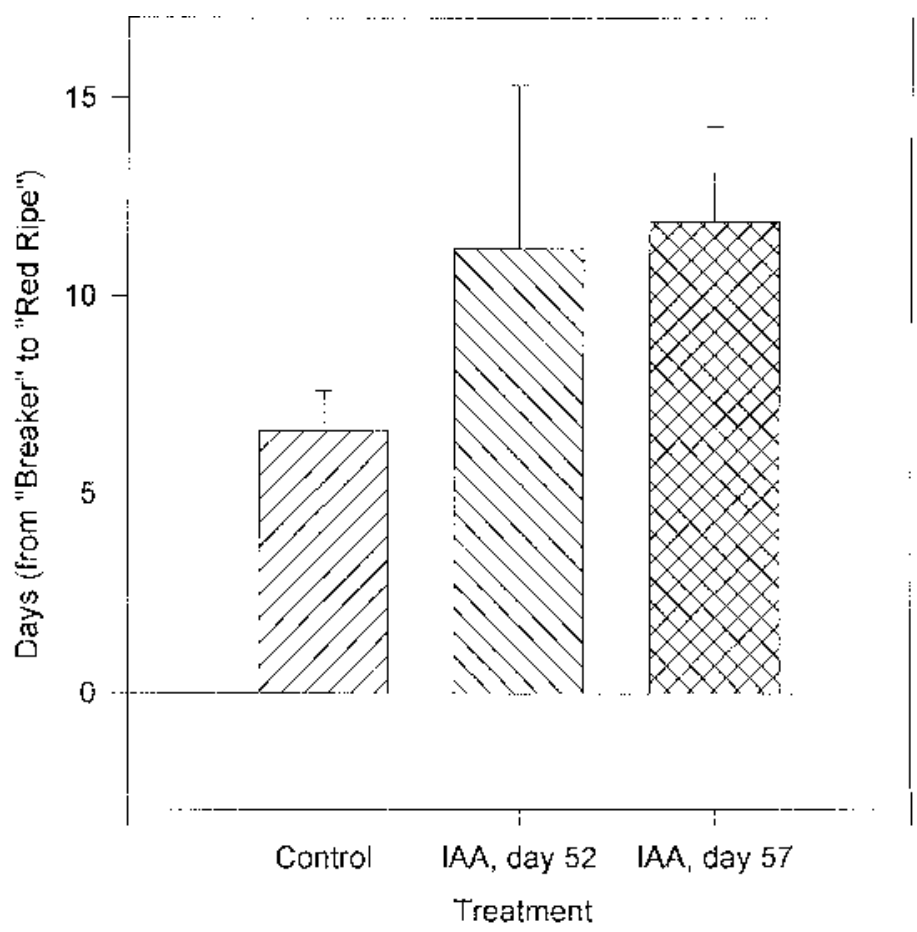

Fig. 3. The effect of added auxin on the time required for in vitro fruit to mature from breaker stage to red ripe stage. 'Ailsa Craig' tomato flowers 3-4 $\mathrm{mm}$ in size were sterilized and planted on agar containing $20 \mathrm{ml}$ of Murashige and Skoog medium with $10^{-4} \mathrm{M}$ IAA in a $50 \mathrm{ml}$ sterile disposable centrifuge tube. After 52 or 57 days, $200 \mu \mathrm{l}$ of $10^{-3} \mathrm{M}$ IAA was added as indicated. Sample size was 24 for control and 12 for each treatment. Error bars indicate SE and the data for the day 52 and day 57 treatments were significantly different from controls at $P=0.05$ and 0.01 , respectively. Data were analyzed using the $t$ test.

senesced without going through the full ripening program. The remainder developed into small red-ripe seedless fruit (Fig. 2).

IAA effects on fruit ripening. The effect of adding IAA on fruit ripening was tested by adding IAA to the culture media of immature and mature green fruit. The amount added was $200 \mu \mathrm{l}$ of a $10^{-3} \mathrm{M}$ IAA solution to the $20 \mathrm{ml}$ of agar medium to give a final concentration of $10^{-5} \mathrm{M}$ IAA. Thus, the added IAA was equal to about $10 \%$ of that which had been in the medium at the start of the experiment for flower initiation. As shown in Fig. 3, the addition of IAA late in the culture period almost doubled the mean time between breaker stage and red-ripe. Treatment did not affect the days to breaker nor did treatment more than 10 days before breaker have any noticeable effect (data not shown). IAA addition to the culture media resulted in a marked increase in internal IAA levels in the breaker stage fruit (Table 1).

\section{Discussion}

Studies with whole fruit of tomato and of other crops have shown that auxin treatments can provide some delay in fruit

Table 1. Indole-3-acetic acid levels in breaker stage (day 62) in vitro tomatoes as determined by gas chromatography-selected ion monitoring-mass spectrometric analysis.

\begin{tabular}{|c|c|}
\hline Treatment & $\begin{array}{l}\text { IAA content } \\
\mathrm{ng} \cdot \mathrm{g}^{-1} \text { fresh } \mathrm{wt}\end{array}$ \\
\hline Control (10 ${ }^{-4}$ M IAA provided at planting only) & $3.4 \pm 1.5$ \\
\hline $\begin{array}{l}\text { Treated ( } 20 \mathrm{ml} \text { of media containing } 10^{-4} \mathrm{M} \text { IAA at } \\
\text { planting and treated with } 200 \mathrm{ml} 10^{-3} \mathrm{M} \text { IAA at day } 57 \text { ) }\end{array}$ & $16.3 \pm 4.2$ \\
\hline
\end{tabular}


ripening and softening (Abdel-Kader et al., 1966; Babbitt et al., 1973; Frenkel and Dyck, 1973; McGlasson et al., 1978; Tingwa and Young, 1975; Vendrell, 1969) and that antiauxins have the reverse effect (Frankel and Haard, 1973). To obtain such results for auxin treatment, however, required vacuum infiltration of IAA into the fruit (Frenkel and Dyck, 1973; Vendrell, 1969; Tingwa and Young, 1975) or prolonged dipping of whole fruit (Abdel-Kader et al., 1966) with a resultant uncertainty in relation to uptake and distribution (Vendrell, 1970). Such techniques usually also require that the fruit be harvested before treatment.

The problems of tissue sections and the difficulties of using whole fruit prompted us to consider alternative approaches. In particular, we desired a system that was suitable for complex labeling studies to establish precursor/product relationships in hormone metabolism, a system in which transgene effects could be studied in isolation from the mother plant, and where normal development and ripening could be studied while hormone levels and metabolism were manipulated. Tissue culture has been used to overcome similar problems in other situations, and the original report by Nitsch (1951) and more recent results reported by Mukai (1985) and Ishida (1993) prompted us to consider such an approach to study fruit development. Based on these earlier studies we developed a system for the mass culture of immature tomato infloresences to produce parthenocarpic fruit.

It is interesting to note that IAA was the only auxin that gave consistent fruit production from cultured flowers. Unlike auxin conjugates and synthetic auxins that provide an auxin source over prolonged culture (Hangarter and Good, 1981), IAA in culture media is more transient and has been reported to be lost from the medium over the first 5-7 days (Dunlap et al., 1986, Dunlap and Robacker, 1988, Stasinopoulos and Hangarter, 1990). This suggests that auxin in the medium may be required for fruit induction but not for organ growth. This hypothesis is also supported by the low IAA content in fruit at the breaker stage (Table 1).

Using the in vitro system it was possible to test the earlier concepts about the role of IAA in fruit ripening. As discussed above, tissue sections respond to high levels of IAA by increased rates of ripening, probably due to ethylene induction. Whole fruit studies had suggested that ripening could be delayed by treatment with moderate levels of IAA. Using in vitro fruit, we have confirmed and extended the earlier observation that tomato fruit undergo a delay in the final steps of fruit ripening if IAA is provided to the fruit at the mature green stage. It is important to note, however, that in this current study the color change accompanying ripening in tomato was the primary criteria for evaluation and we do not yet know if other changes, such as acidity, texture, and flavor are similarly changed by auxin treatment. We do not yet know the relationship between ripening of in vitro fruit and those that normally develop on the plant. Comparisons between these to situations should be done carefully. IAA levels in the in vitro fruit, however, were similar to that reported by Buta and Spaulding (1994) for tomato pericarp from vine grown fruit at the same stage of ripening.

\section{Literature Cited}

Abdel-Kader, A.S., L.L. Morris, and E.C. Maxie. 1966. Effect of growthregulating substances on ripening and shelf-life of tomatoes. Hortscience 1:90-91.

Babbitt, J.K., M.J. Powers, and M.E. Patterson. 1972. Effects of growthregulators on cellulase, polygalacturonase, respiration, color, and texture of ripening tomatoes. J. Amer. Soc. Hort. Sci. 98:77-81.

Bangerth, F. 1983. Hormonal and chemical preharvest treatments that influence postharvest quality, maturity, and storeability of fruit, p. 331-
354. In: M. Lieberman (ed.). Post-harvest physiology and crop preservation. Plenum, New York.

Brady, C.J. 1987. Fruit ripening. Annu. Rev. Plant Physiol. 38:155-178.

Buta, J.G. and D.W. Spaulding. 1994. Changes in indole-3-acetic acid and abscisic acid levels during tomato (Lycopersicon esculentum Mill.) fruit development and ripening. J. Plant Growth Regulat. 13:163-166.

Campbell, A.D., M. Huysamer, H.U. Stotz, L.C. Greve, and J.M.Labavitch. 1990. Comparison of ripening progresses in intact tomato fruit and excised pericarp discs. Plant Physiol. 94:1582-1589.

Castillo, F.J. 1986. Extracellular peroxidases as markers of stress?, p. 419-426. In: H. Greppin, C. Penel, and T. Gaspar (eds.). Molecular and physiological aspects of plant peroxidases. Univ. of Geneva, Switzerland.

Catalá, C., A. Östin, J. Chamarro, G. Sandberg, and A. Crozier. 1992. Metabolism of indole-3-acetic acid by pericarp discs from immature and mature tomato (Lycopersiconesculentum Mill.). Plant Physiol. 100:14571463.

Catalá, C., A. Crozier, and J. Chamarro. 1994. Decarboxylative metabolism of $\left(1{ }^{1}-{ }^{14} \mathrm{C}\right)$ indole-3-acetic acid by tomato pericarp discs during ripening. Planta 193:508-513.

Chen, K-H., A.N. Miller, G.W. Patterson, and J.D. Cohen. 1988. A rapid and simple procedure for purification of indole-3-acetic acid before GCSIM-MS analysis. Plant Physiol. 86:822-825.

Cohen, J.D. 1984. Convenient apparatus for the generation of small amounts of diazomethane. J. Chromatography. 303:193-196.

Dunlap, J.R., S. Kresovich, and R.E. McGee. 1986. The effect of salt concentration on auxin stability in culture media. Plant Physiol. 81:934936.

Dunlap, J.R. and K.M. Robacker. 1988. Nutrient salts promote lightinduced degradation of indole-3-acetic acid in tissue culture media. Plant Physiol. 88:379-382.

Frenkel, C. and R. Dyck. 1973. Auxin inhibition of ripening in Bartlett pears. Plant Physiol. 51:6-9.

Frenkel C. and N.F. Haard. 1973. Initiation of ripening in Bartlett pears with an antiauxin (p-chlorophenoxy)isobutyric acid. Plant Physiol. 52:380-384

Gamborg, O.L. and J.P. Shyluk. 1981. Nutrition, media and characteristics of plant cell and tissue cultures, p. 21-44. In: T.A. Thorpe (ed.). Plant tissue cultures, Academic Press, New York.

Gouble, B. and P. Soudain. 1993. Charactérisation de la maturation de disques de péricarpe de tomate en relation avec l'effet de blessure. C.R. Acad. Sci., Paris Sciences de la vie 316:1240-1244.

Hangarter, R.P. and N.E. Good. 1981. Evidence that IAA conjugates are slow-release sources of free IAA in plant tissues. Plant Physiol. 69:14241427.

Ishida, B.K. 1991. Developmental regulation is altered in the calyx during in vitro ovary culture of tomato. Plant Cell 3:219-223.

Ishida, B.K., E.A. Baldwin, R.G. Buttery, S.H. Chui, and L.C. Ling. 1993. Flavor volatiles, sugar and color development in ripening in vitrocultured tomato fruit and calyx. Physiol. Plant 89:861-867.

Kokkinakis, D.M. and J.L. Brooks. 1979. Hydrogen peroxide-mediated oxidation of indole-3-acetic acid by tomato peroxidase and molecular oxygen. Plant Physiol. 64:220-223.

McGlasson, W.B., N.L. Wade and J. Adato. 1978. Phytohormones and fruit ripening, p. 447-493. In: D.S. Letham, P.B. Goodwin and T.J. Higgins (eds.). Phytohormones and related compounds. A comprehensive treatise, vol. 2. Elsevier, Amsterdam.

Miller, A.N. 1990. Changes in auxin concentration in developing fruits and seeds of non-endospermic seed species. Plant Growth Regulat. Soc. Amer. Quarterly 18:166-176.

Miller, A.N., C.S. Walsh, and J.D. Cohen. 1987. Measurement of indole3-acetic acid in peach fruits (Prunus persica L. Batsch cv. Redhaven) during ripening. Plant Physiol. 84:491-494.

Mukai M.K. 1985. Metodo de cultura in vitro de frutos de tomate para estudos pós-colheita. Hort. bras. 3:27-28.

Nitsch, J.P. 1951. Growth and development in vitro of excised ovaries. Amer. J. Bot. 38:566-577.

Normanly, J., J.D. Cohen, and G.R. Fink. 1993. Arabidopsis thaliana auxotrophs reveal a tryptophan-independent biosynthetic pathway for 
indole-3-acetic acid. Proc. Natl. Acad. Sci. USA 90:10355-10359.

Normanly, J., J.P. Slovin, and J.D. Cohen. 1995. Rethinking auxin biosynthesis and metabolism. Plant Physiol. 107:323-329.

Nowacki, J., J.D. Cohen, and R.S. Bandurski. 1978. Synthesis of ${ }^{14} \mathrm{C}-$ indole-3-acetyl-myo-inositol. J. Labelled Compounds and Radiopharm. 15:325-329.

Riov, J. and F. Bangerth. 1992. Metabolism of auxin in tomato fruit tissue. Formation of high molecular weight conjugates of oxindole-3-acetic acid via the oxidation of indole-3-acetylaspartate. Plant Physiol. 100:1396-1402.

Slovin, J.P. and J.D. Cohen. 1993. Auxin metabolism in relation to fruit ripening. Acta Hort. 329: 84-89.

Stasinopoulos, T.C. and R.P. Hangarter. 1990. Preventing photochemistry in culture media by long-pass light filters alters growth of cultured tissues. Plant Physiol. 93:1365-1369.

Tam Y.Y., J.P. Slovin, and J.D. Cohen. 1995. Selection and characterization of $\alpha$-methyltryptophan resistant lines of Lemna gibba showing a rapid rate of indole-3-acetic acid turnover. Plant Physiol. 107:77-85. Tingwa, P.O. and R.E. Young. 1975. The effect of indole-3-acetic acid and other growth regulators on the ripening of avocado fruits. Plant Physiol. 55:937-940.

Vendrell, M. 1969. Reversion of senescence: Effects of 2,4dichlorophenoxyacetic acid and indoleacetic acid on respiration, ethylene production, and ripening of banana fruit slices. Austral. J. Biol. Sci. 22:601-610.

Vendrell, M. 1970. Relationship between internal distribution of exogenous auxins and accelerated ripening of banana fruit. Austral. J. Biol. Sci. 23:1133-1142.

Wright, A.D., M.B. Sampson, M.G. Neuffer,L. Michalczuk, J.P. Slovin, and J.D. Cohen. 1991. Indole-3-acetic acid biosynthesis in the mutant maize orange pericarp, a tryptophan auxotroph. Science 254:998-1000.

Yunovitz, H. and K.C. Gross. 1994. Delay of tomato fruit ripening by an oligosaccharide $\mathrm{N}$-glucan. Interactions with IAA, galactose, and lectins. Physiol. Plant. 90:152-156. 